\title{
Les mondes inversés
}

Seconde livraison de la revue de l'année 2020, ce numéro paraît dans un contexte international malheureusement toujours bouleversé par la pandémie de Covid-19. Nous réitérons notre soutien aux personnes ayant été touchées par le virus et ses conséquences.

Dans ce lourd contexte, nous sommes néanmoins heureux d'annoncer l'indexation de CF dans la base de données EBSCO. En complément de notre indexation au DOAJ, cette association permettra à CF de toucher un plus large public et d'offrir aux auteur.e.s une plus grande visibilité. Afin d'élargir les champs d'études et les perspectives, nous avons également souhaité ouvrir la revue à des collaborations éditoriales (voir la section Annonces sur le site de la revue). Deux numéros sont d'ores et déjà envisagés pour 2021 et 2022.

Le numéro 6.3 conclut l'année 2020 sur le thème de l'inversion. De la représentation des antipodes à l'inversion des rôles en passant par l'anastrophe, les jeux de miroir, les retournements de situation ou l'inversion de l'Histoire, la littérature (au sens large) et le cinéma mettent à profit un trope qui, semble-t-il, ne s'épuise pas comme le montrent Aux Etats-Unis d'Afrique (2005) d'Abdourahman Waberi, Jacky au royaume des filles (2014) de Riad Sattouf ou encore Je ne suis pas un homme facile (2018) d'Eléonore Pourriat.

Cette actualité de l'inversion semble renvoyer à une pertinence propre à la mise en intrigue de l'expérience humaine mais aussi aux lois physiques naturelles - l'inversion des pôles magnétiques de la Terre le rappelle tous les jours. Pour les anthropologues, l'histoire d'Asdiwal - consignée par Franz Boas et passée à la moulinette structuraliste par Claude Lévi-Strauss - reposerait en partie sur ce trope. A moins que, comme semble le montrer les critiques de cette célèbre étude, la critique occidentale n'ait projeté sur le patrimoine culturel du peuple Tsimshian de la Colombie-Britannique sa propre vision du monde. Moins sujettes à controverse, les études classiques d'Ernst Robert Curtius et de Mikhaïl Bakhtine sur des corpus occidentaux ont mis en évidence le caractère structurant de l'inversion ainsi que sa fonction subversive et en même temps régulatrice. De son côté, Henri Bergson s'est attaché aux effets de l'inversion linguistique dans son étude sur le rire et l'humour au travers des jeux de mots. Dans tous les cas, l'inversion signifie en déjouant les attentes liées à la manifestation d'un présupposé culturel : l'inversion surprend en créant une distance, un écart vis-à-vis d'une norme. Ainsi la valeur subversive de l'ironie s'exprime par une inversion, un renversement de la formulation attendue d'un message. ${ }^{1}$

Toutefois, dans le cas de la France du XVIe au XIXe siècle, la valeur subversive de l'inversion ne serait qu'apparente dans la mesure où la représentation de l'envers s'appuie sur une vision statique du monde qui garantit l'impossibilité de la réalisation de l'inversion (Bercé 15). Ainsi une lecture de l'illustration retenue pour la couverture de ce numéro (un détail d'une image d'Epinal tiré de la planche intitulée Le Monde renversé) privilégie l'idée qu'en

\footnotetext{
${ }^{1}$ Outre l'ouvrage bien connu de Philippe Hamon (L'ironie littéraire. Essai sur les formes de l'écriture oblique. Paris : Hachette université, 1996), citons également celui de Patrick Neiertz, Lumières obliques. Ironie et dialogues au XVIIIe siècle. Paris : Champion, 2012.
} 
montrant ce qui est jugé impossible, on renforcerait en fait des certitudes partagées. L'inversion cosmique serait ici rassurante car elle conforterait paradoxalement la position initiale, dominante et conservatrice (Bercé). Sans nuire à la pertinence de cette réflexion, il semble cependant délicat de restreindre l'imaginaire d'une époque à la seule lecture de vignettes car l'inversion ne se manifeste pas seulement dans les images dites populaires ou les textes d'historiens du XVIe siècle. L'inversion n'est pas seulement un objet de représentation, elle est aussi mode de représentation. En effet, cette inversion cosmique où "les maisons éclairent le soleil et la lune" pourrait également être vue comme un questionnement portant sur la pertinence du point de vue. C'est ce qu'on voit au XVIIIe siècle avec les Lettres persanes de Montesquieu qui inversent, comme chacun sait, la perspective alors traditionnellement eurocentrique de l'Européen observateur et de l'Autre (non-Européen) observé pour livrer une satire des croyances et des institutions. Et l'auteur français ne représente pas ici l'impossible, si l'on se souvient qu'il existe aussi un "ailleurs de l'autre" (Le Blanc) que matérialisent les récits de voyageurs non-européens en Europe comme le Seyahatnâme d'Evliya Çelebi qui a parcouru l'Europe au XVIIe siècle (Apostolou), ou encore les récits des ambassades chinoises en France publiés dès le XVIIe siècle comme la Harangue faite à sa Majesté par les Ambassadeurs du Roy de Siam. L'inversion du regard persan, même fictive, est dans ce cas-là une réalité. ${ }^{2}$ L'inversion compterait donc bien au moins deux fonctions : l'une régulatrice et l'autre réellement subversive.

Enfin, il faut signaler que l'inversion n'est pas limitée à la version simplement contraire ou opposée d'une action, d'un état ou d'un dire. Elle peut aussi, par l'idée du renversement, être synonyme de la fin d'un état, d'une situation et par-là, renvoyer à l'idée du changement, voire du commencement. ${ }^{3}$

L'inversion interroge, interpelle et les textes réunis ici permettent d'éclairer ce thème et ses fonctions au prisme d'un corpus littéraire francophone récent. Ce numéro s'ouvre sur «Les mondes inversés de Gherasim Luca - Étude de trois poèmes » de Charlène Clonts. Poète roumain francophone, Gherasim Luca donne à voir et à entendre un imaginaire qui renverse les codes de la langue et de la représentation, hors du champ des signifiants et des signifiés. La violence oxymorique de la poésie de Lucas perturbe la relation entre le signifiant et le signifié et dépeint un imaginaire à la limite du représentable. Perturbatrice du sens, au singulier comme au pluriel, la poésie de Luca, inclassable, force ainsi son lecteur à une remise en question de son rapport au langage, à l'identité et au monde.

C'est également d'une remise en question de notre rapport à l'identité et au monde dont il question dans l'article de Jennifer Boum Make « Penser audelà du binarisme: Étude des stratégies de reconnaissance et de ré/conciliation des altérités dans Aux États-Unis d'Afrique d'Abdourahman Waberi». Boum Make s'intéresse au binarisme inversé du monde dépeint par Abdourahman Waberi dans Aux États-Unis d'Afrique, où le continent africain incarne le pôle géographique, culturel, politique et économique du monde tandis que l'Euramérique se voit appauvrie, dans tous les sens du terme. Si cette inversion

\footnotetext{
${ }^{2} \mathrm{Si}$ l'on veut rester dans le domaine de la fiction et du cosmos, songeons aussi au Micromégas de Voltaire et à sa critique des vérités absolues de la religion.

${ }^{3} \mathrm{Ce}$ qui renvoie au thème du numéro inaugural de cette revue.
} 
semble reproduire les schémas binaires sous-tendant les rapports de force coloniaux, le nomadisme de la protagoniste, Maya/Malaïka, en quête de ses origines, transcende le schéma colonial du "Nous-contre-Eux". A une altérité coloniale mortifère, Waberi proposerait plutôt un modèle de ré/conciliation des particularismes locaux, dépassant une pensée binaire, faisant chuter les rapports de dominations.

La contribution de Molleen Shilliday "Les Inversions de l'héritage littéraire entre Eric-Emmanuel Schmitt et Romain Gary" interroge la filiation littéraire et humaniste entre Eric-Emmanuel Schmitt et Romain Gary. Selon Shilliday, le roman de Schmitt, Monsieur Ibrahim et les fleurs du Coran, est bien plus qu'une simple réécriture de La vie devant soi de Gary et dépasse le simple clin d'œil complice à son lecteur avisé. Certes, Schmitt joue de l'inversion - chez Gary un enfant musulman est élevé par une vieille femme juive ; chez Schmitt un enfant juif se lie d'amitié avec un vieil homme arabe mais il entre surtout en dialogue avec Gary pour créer un roman à quatre mains marqué par l'humour et prônant l'amour, l'empathie et l'humanité.

La section thématique de ce numéro se conclut avec l'article de Zvesdana Ostojic, « Le verso de la p(l)age de Camus: intertextualité militante dans Meursault, contre-enquête », où elle propose une lecture du roman Meursault, contre enquête de l'écrivain algérien Kamel Daoud, prix Goncourt du premier roman 2015. Selon Ostojic, le roman de Daoud procède à un renversement méthodique de l'œuvre de Camus (de genre et de point de vue) et se construit selon une intertextualité plurielle (linguistique, littéraire et culturelle). Le roman de Daoud fait le choix du genre policier, genre mineur, pour procéder à la déconstruction et à la reconstruction de l'oeuvre de Camus en lui apposant un verso subversif où le colonisé prend enfin parole.

Dans la section Varia, l'article de Karel Plaiche «L'ombre d'Imana ou "l'expérience du dire" du génocide au Rwanda », s'intéresse à l'utilisation du genre de récit de voyage pour rendre compte du génocide des Rwandais tutsi dans le roman de l'Ivoirienne Véronique Tadjo. Selon Plaiche, le choix du genre du récit de voyage s'avère judicieux de la part de Tadjo, témoin indirect de la violence extrême du génocide, se rendant au Rwanda quatre ans après les faits. La confrontation aux événements et aux témoignages s'écrit par une alliance entre la violence extrême des faits avérés et la crise du langage: seuls l'hybridité et le fragmentaire sont à même de rendre compte des faits. L'écriture de Tadjo est sous-tendue par une " politique de non-fixité et de non-unicité » où de nouveaux types d'écritures doivent être adoptés afin de rendre compte de l'impensable et de l'innommable. Le mélange de genres, le langage fragmenté et fragmentaire s'allient parfaitement au sujet douloureux et (in)humain de ce qui est « un mémoire de devoir».

Pour leur soutien, nous tenons aussi à remercier Christiane Achour, Kusum Aggarwal, Veronic Algeri, Markus Arnold, Viviane Azarian, Cécile Bertin, Didier Coureau, Ninon Chavoz, Guy Dugas, Pierre Halen, Nicolas Hauck, Karen Ferreira-Meyers, Serge Martin, Sofija Perovic, Antony Soron et Sylvie Triaire.

Les éditeurs 


\section{Bibliographie}

Adams, John W. "Dialectics and Contingency in "The Story of Asdiwal": An Ethnographic Note. The Unconscious in Culture : The Structuralism of Claude Lévi-Strauss in Perspective. Dir. Ino Rossi. New York, Dutton. pp. 170-178.

Apostolou, Irini. «La représentation de soi et de l'autre dans le Seyâhatnâme, ou Livre des voyages d'Evliya Celebi : étude comparative avec les voyageurs français du xvii ${ }^{\mathrm{e}}$ siècle. " L'ailleurs de l'autre : Récits de voyageurs extra-européens. Dir. Claudine Le Blanc et Jacques Weber. Rennes : Presses universitaires de Rennes, 2009. pp. 125-134. DOI: https://doi.org/10.4000/books.pur.26327.

Bakhtine, Mikhaïl. L'Euvre de François Rabelais et la culture populaire au Moyen Age et sous la Renaissance. Paris : Gallimard, 1970.

Bercé, Yves-Marie « La fascination du monde renversé dans les troubles du XVIe siècle. " L'image du monde renversé et ses représentations littéraires et paralittéraires de la fin du XVIe siècle au milieu du XVIIe. Dir. Jean Lafond et Augustin Redondo. Paris : Vrin, 1979. Pp. 9-15.

Bergson, Henri. Le Rire: essai sur la signification du comique, Paris, Félix Alcan 1900.

Curtius, Ernst Robert. La Littérature européenne et le Moyen Âge latin. Paris : Presses Universitaires de France, 1956.

Harangue faite à sa Majesté par les Ambassadeurs du Roy de Siam. Paris : Sébastien Marbre-Cramoisy, 1687.

Le Blanc, Claudine. «Introduction. » L'ailleurs de l'autre : Récits de voyageurs extra-européens. Dir. Claudine Le Blanc et Jacques Weber. Rennes : Presses universitaires de Rennes, 2009. pp. 11-15. DOI: https://doi.org/10.4000/books.pur.26303.

Lévi-Strauss Claude. « La geste d'Asdiwal. » École pratique des hautes études, Section des sciences religieuses. Annuaire 1958-1959. 1957. DOI : https://doi.org/10.3406/ephe.1957.17956. pp. 3-43.

Thomas L.L, Kronenfeld, J.Z. et Kronenfeld D.B. « Asdiwal crumbles: a critique of Lévi-Straussian myth analysis. » American Ethnologist, vol. 3, no. 1, 1976, pp. 147-173.

\section{Référence de l'illustration}

Le monde renversé (Ire planche). De la fabrique de Pellerin, imprimeur-libraire à Epinal [1829]. Bibliothèque nationale de France. https://gallica.bnf.fr/ark:/12148/btv1b55002032h 